\title{
L'Accademia Gioenia al tempo della "Spagnola"
}

\author{
M. Alberghina ${ }^{1 *}$ \\ ${ }^{1}$ Accademia Gioenia di Catania, Catania, Italy
}

\begin{abstract}
Riassunto
Si riporta un esame critico dell'attenzione rivolta alla "Spanish flu" o influenza spagnola, comparsa all'inizio del XX secolo come malattia letale, ad opera di soci dell'Academia Gioenia di Catania. La grande pandemia d'infezione polmonare, secondo gli studiosi, fu definita come "uno dei più grandi nemici dell'uomo". Tuttavia, le caratteristiche della ricorrente pandemia che colpì la maggioranza della popolazione della Sicilia nell'autunno del 1918, l'agente eziologico, le misure sociali e i rimedi sanitari approntati al suo esordio sono stati sorprendentemente poco esaminati e discussi in adunanze accademiche e anche nelle pubblicazioni apparse nelle due riviste edite dall'Accademia.
\end{abstract}

Parole chiave: influenza spagnola, pandemia, Accademia Gioenia, Catania.

\section{Summary}

\section{Gioeni's Academy in the time of the "Spanish flu"}

The critical analysis here undertaken deals with the attention devoted to "Spanish flu", broken out at the beginning of the Twentieth Century as an influenza disease, by the associates of Gioeni's Academy in the Sicilian city of Catania. The great pandemic of the pulmonary infection, according to the scholars was defined as "one of the greatest enemies of man". However, the characteristics the etiological agent, the social care systems as well as sanitary remedial actions in the autumn of 1918, when outbreaks occurred, surprisingly, were little examined and discussed in the academic meetings and even in publications appeared in two scientific journals edited by Gioeni's Academy.

Keywords: Spanish flu, pandemic, Gioeni's Academy, Catania, scientific journals.

\footnotetext{
*E-mail: malber@unict.it
} 


\section{Introduzione}

Nell'Ottocento Il termine "epidemia" era molto ricorrente e presente, associato a patologie diffuse e frequenti come la peste e il colera. Quest'ultima malattia era molto attuale e ciclica in quel secolo e per essa rimaneva un persistente ricordo e un'ampia letteratura storica, clinica ed epidemiologica che descriveva gli elementi del contagio. L'elenco degli episodi epidemici della malattia era lungo: epidemia di colera a Napoli e in Sicilia nel 1836-37, ancora a Napoli e in Sicilia nel 1854-55, in Sicilia nel 1867, la diffusa pandemia in Europa ed Asia tra il 1881 e il 1896 che toccò Napoli nel 1884, Palermo nel 1885 e tutta la Sicilia nel 1887, le epidemie in Germania e in Francia nel 1892, a Napoli e Palermo nel 1910-1911 (Maggiore Perni, 1894; Aymard, 1975).

Le pandemie d'influenza o "grippe" riscuotevano meno attenzione a causa del fraintendimento e della confusione conoscitiva con altre malattie febbrili meno mortali come l'influenza stagionale, la polmonite, la tubercolosi, la meningite, il "catarro epidemiale", le infezioni respiratorie acute. L'attenzione si risvegliò in seguito alla presenza di un'epidemia di influenza più consistente e preoccupante che si ebbe in Sicilia nel periodo 1889-1890 (Vicentini et al., 2015). La mortalità nell'isola e a Catania fu lieve, mentre la malattia si mostrò epidemica e più letale a San Pietroburgo, Parigi, Berlino (l'influenza "russa"), con recidive nel periodo 1891-1895. Tra le tante vittime eccellenti di quel periodo vi furono la matematica e scrittrice russa Sofja Vasilevna Kovalevskaja, il medico londinese Henry Gawen Sutton e l'antropologo e biologo marino francese Jean L. A. de Quatrefages de Bréau. Quest'ultimo nella tarda primavera del 1844, durante una spedizione nel Mediterraneo, aveva visitato Palermo, le coste della Sicilia occidentale, Castellammare, Segesta, Trapani, le tonnare di Favignana insieme a H. Milne Edwards e C. É. Blanchard, componenti di una Commissione scientifica francese (de Quatrefages, 1857).

Successivamente si ebbe l'avvento dell'influenza che sconvolse il mondo (Kolata, 2000; Winter, 2010; Spinney, 2018), chiamata "spagnola" perché fu inizialmente descritta nella penisola iberica, ma in realtà fu portata nel vecchio continente dalle truppe statunitensi che a partire dall'aprile 1917 confluirono in Francia a seguito all'entrata degli Stati Uniti nel primo conflitto mondiale. È proprio dalle biopsie di alcuni militari americani deceduti per l'influenza e da campioni di tessuto polmonare conservato in abitanti dell' Alaska dal permafrost che i ricercatori hanno potuto isolare il virus e studiarlo alla luce delle attuali conoscenze. Altri esperti, invece, hanno sostenuto l'origine asiatica della malattia. Il virus, originatosi nei sobborghi malsani delle metropoli cinesi, sarebbe stato veicolato nel globo dai lavoratori asiatici diretti in Francia passando dal Nord America. Recentemente il virologo John Oxford (2001) ha ipotizzato che il virus della spagnola avrebbe avuto origine nel campo militare inglese di Étaples, non distante dal dipartimento della Somme. Il virus influenzale più tardi identificato era il sottotipo H1N1, derivato o mutato da un virus aviario.

In otto mesi, tra il settembre 1918 e l'aprile 1919, la pandemia di influenza spagnola colpì metà della popolazione mondiale uccidendo almeno 50 milioni 
di persone: circa 375.000 in Italia (ma alcuni sostengono 600.000, più di quelle che il nostro Paese aveva pianto alla fine della Grande Guerra). La regione che ebbe in assoluto il numero maggiore di morti fu la Lombardia (36.653), seguita dalla Sicilia (29.966). Nella "Relazione 2017" del medico provinciale cav. Nicolò Consoli al Consiglio sanitario di Catania, dove si riferisce sulla continuazione della profilassi del vaiolo mediante vaccinazione e della campagna antimalarica, nulla è segnalato su una possibile epidemia di influenza (Sicilia, giornale politico-quotidiano di Catania, 1918, anno XVIII, n. 202, 27-28 luglio). Improvvisamente nel 1918, a Catania e nella sua provincia si ebbero due picchi epidemici, nei mesi maggio-giugno e settembre-ottobre. Questo secondo picco si mostrò più grave. Furono colpiti individui, anche molto giovani, in istituti di assistenza, reclusori, convitti, ospizi e la popolazione di tutti i quartieri.

Il primo articolo sull'epidemia scoppiata in città, dal titolo singolare " $\mathrm{La}$ malattia di moda. I sintomi e la profilassi", dove sono incluse alcune norme di comportamento sociale, apparve sul giornale Corriere di Catania n. 244 (Cronaca di Catania, 11 settembre 1918). In contemporanea, sul giornale Sicilia (1918, anno XVIII, n. 244, 11-12 settembre) comparve un articolo di cronaca che titolava " $\mathrm{La}$ malattia del giorno", dove erano consigliate molte norme di profilassi. Il sindaco Antonio Sapuppo Asmundo ordinò la disinfestazione dei locali pubblici il successivo 19 settembre (Sicilia, 1918, anno XVIII, n. 253, 20-21 settembre).

Un articolo di cronaca dal titolo Per la salute pubblica che riferisce di una riunione delle Autorità in Prefettura, dove era stata definita una linea di condotta da tenere senza allarmismi e indicate disposizioni per la chiusura dei teatri e cinematografi in locali chiusi, fu pubblicato dal giornale Sicilia (1918, anno XVIII, n. 256, 23-24 settembre). Anche sul giornale Corriere di Catania di mercoledì 25 settembre 1918 apparve un articolo di cronaca dal titolo "L'influenza estiva o febbre spagnuola. Come si diffonde - Come si combatte".

Il quotidiano palermitano L'Ora (attivo dal 1900), nella parte riguardante la cronaca di Catania, scriveva: «Le notizie di oggi sono però migliori. Le autorità continuano nelle misure sanitarie ed igieniche atte a fronteggiare il morbo, e a provvedere perché gli ammalati non siano privati degli alimenti necessari.» In effetti il 30 settembre 1918, giorno della pubblicazione di quest'articolo, a Catania si registrano 85 decessi (Tognotti, 2015), il 2 ottobre 82 morti. Fino alla metà del mese la media fu di 30 decessi al giorno, cifra scemata nelle settimane successive (Cronache del giornale Sicilia, anno XVIII, ottobre 1918). A Palermo, la mortalità media giornaliera era di 20-30 persone, ma il 25 settembre si ebbero 177 morti. A Napoli, il 7 ottobre morirono 256 persone (Tognotti, 2015).

A Catania furono rinviati gli esami all'Università, mentre la regolare apertura dell'Anno accademico e delle scuole medie ed elementari cittadine si ebbe solo all'inizio di novembre. Il sindaco, oltre a potenziare l'Ufficio municipale d'Igiene, decise di costituire un Corpo consultivo di medici formato da docenti dell'Università. Esso comprendeva i professori Gesualdo Clementi, Raimondo Feletti, Giuseppe D'Abundo, Filippo Foderà, Salvatore Rindone e Nunzio Di Stefano (Corriere di Catania, Cronaca di Catania, 28 settembre 1918, n. 261; Sicilia, 1918, anno XVIII, n. 264, 1-2 ottobre). Nella riunione al Municipio del 30 settem- 
bre la Consulta si limitò ad emette una serie di consigli sanitari e a raccomandare il rispetto di norme igieniche contro l'epidemia in atto (disinfezione dei locali, fornitura di sapone agli indigenti, istituzione di locali per il ricovero di ammalati, divieto di assembramenti).

Ai primi di novembre fu pubblicata la notizia che l'on. Giuseppe De Felice, presidente del Consiglio Provinciale di Catania, si trovava ammalato di febbre a Palermo (Corriere di Catania, Cronaca di Catania) e che il rettore on. Giuseppe Majorana si era «ammalato dell'epidemia del giorno e che ora, però, trovasi in via di completa guarigione» (Sicilia, 1918, anno XVIII, n. 297, 3-4 novembre).

Un trafiletto giornalistico riportava che il prof. Vittorio Ascoli, clinico medico di Roma, aveva pubblicato in una intervista apparsa sul Tempo di Roma la sua opinione sull'influenza spagnuola, sulle cure e sulla sua durata (Corriere di Catania, Cronaca di Catania, 10 ottobre 1918, n. 273). Un altro trafiletto dal titolo "Il siero della grippe, scoperto" riportava una notizia piuttosto inverosimile da Roma secondo la quale il corrispondente parigino del giornale romano L'Idea Nazionale telegrafava che «Si annuncia che a Tourot (!?) è stato scoperto nel laboratorio di quella Università il siero preventivo della grippe comunemente detta febbre spagnuola. Centomila dosi sono già state distribuite» (Corriere di Catania, Cronaca di Catania, giovedì 24 ottobre 1918, n. 287). Le fake news volavano anche a quel tempo, sebbene circoscritte al solo ambito giornalistico.

Quasi alla fine della pandemia, i professori Luigi Mangiagalli, Luigi Devoto, Serafino Belfanti e Emilio Viganò organizzarono a Milano una riunione dal carattere associativo e pratico. Gli autori si dichiararono scettici circa il ruolo eziologico del bacillo di Pfeiffer che molti indicavano come il microrganismo responsabile della malattia (Atti, 1919). Tale bacillo si rivelò non avere nulla a che fare con l'influenza pandemica, ma vi fu inizialmente associato dal dott. Richard Pfeiffer, allievo di Robert Koch all'Istituto per le malattie infettive della Humboldt-Universität di Berlino. Egli aveva annunciato nel 1892, all'indomani dell'ultima grande ondata pandemica, di aver isolato nel muco nasale di un malato un bacillo ritenuto l'agente causale dell'influenza. Pfeiffer fu docente all'Università di Königsberg dal 1909 e nel 1925 descrisse meglio il coccobacillo Haemophilus influentiae, il microrganismo effettivamente da lui isolato nel 1892, causa di infezioni delle vie respiratorie e meningiti. La natura virale dell'influenza nell'uomo fu scoperta solo nel 1933, in Inghilterra (Smith et al., 1933).

\section{Disinteresse verso l'epidemia influenzale}

Sorprendentemente sull'epidemia di influenza spagnola nessuna notizia è riportata nei verbali delle Sedute e del Consiglio di Amministrazione dell'Accademia Gioenia nel periodo 1918-1920. Nessun documento d'archivio fornisce ai soci e ai lettori informazioni "in tempo reale" sull'entità e sulla gravità dei focolai locali (Archivio Storico Accademia Gioenia, voll. 58, 59, 267). Non risulta nessuna segnalazione degli studi eziologici in corso in tutto il mondo contemporaneo In quel periodo era Presidente dell'Accademia Annibale Riccò, professore di 
astrofisica che lavorava all'Osservatorio astrofisico-etneo in locali appartenuti al monastero dei Padri Benedettini; vice-presidente era il prof. Gesualdo Clementi, clinico chirurgo universitario all'Ospedale Santa Marta in via Lincoln. La conduzione e le attività del sodalizio seguirono l'iter consueto, fatto di adunanze, consigli di amministrazione, cura delle pubblicazioni.

Nelle due pubblicazione seriali dell'Accademia, gli Atti e il Bollettino, furono pubblicati cinque articoli sull'argomento:

- Angelo Petrone, "Sulla cosidetta Febbre spagnuola" (Petrone, 1918). Pugliese di nascita, Petrone era professore ordinario di Anatomia patologica, già Preside della Facoltà di Medicina e Chirurgia, socio effettivo dell'Accademia dal 1890. Era quasi alla conclusione della sua carriera, avvenuta nel 1920. Nell'articolo dichiara che intende attenersi ai fatti e non alle "parole, parole, parole" nei discorsi che si udivano in relazione all'epidemia. Raccomanda linee di comportamento ed elenca i segni clinici della malattia (polmonite, catarro bronchiale). Si interroga sull'agente causale della malattia, esprime il parere che si tratti di una «simbiosi batterica» e si lancia in azzardati consigli terapeutici (uso di piramidone, bicloruro di mercurio, purganti).

- Salvatore Previtera, medico dell'Ufficio d'Igiene del Comune di Catania, "Sull'epidemia d'influenza in Catania nel 1918" (Previtera, 1919). L'articolo è un lungo estratto della Relazione presentata al Sindaco e al Prefetto di Catania il 27 gennaio 1919. È un prezioso lavoro analitico in cui sono riportate note cliniche ed epidemiologiche, tabelle di mortalità generale per età, sesso, professione (registra un totale di 1.805 morti a fine epidemia), dove lancia giudizi negativi sulla scarsità delle misure sanitarie adottate dalle autorità, con l'intento di lasciare traccia per statistiche e studi futuri.

- Guido Izar, "Acetonuria nell'influenza" (Izar, 1920). Il prof. Izar (1883-1967) era aiuto e allievo del prof. Maurizio Ascoli all'Istituto di Patologia speciale medico-dimostrativa dell'Ospedale Vittorio Emanuele II. Fu a Catania dal 1910 al 1926, poi si trasferì a Messina e quindi a Siena come clinico medico. Fu socio corrispondente dell'Accademia dal 1922 al 1940, poi corrispondente non residente da Siena. Nell'articolo si limita a segnalare il referto laboratoristico (presenza di acetone) rilevato in alcuni casi di influenza.

- Salvatore Aradas, "L'influenza. Note cliniche ed osservazioni critiche" (Aradas, 1919-1920). Figlio dello zoologo Andrea Aradas, era medico dell'Ospedale Vittorio Emanuele II e socio ordinario dell'Accademia dal 1887. Nell'articolo sottolinea il confusionismo clinico, terapeutico e laboratoristico sulla malattia influenzale sopravvenuta e si interroga sul vero agente patogeno (natura batterica o virale filtrabile). Formula un auspicio sui progressi della batteriologia «per evitare e proteggerci da pur troppo facili successive invasioni».

- Antonio Fagiuoli, "Sui rapporti tra tubercolosi e influenza" (Fagiuoli, 1920). Allievo e assistente del prof. Maurizio Ascoli, libero docente di Patologia 
medica, farà parte della Facoltà di Medicina nel 1922. Nell'articolo sostiene che la pandemia aveva aperto questioni inerenti ai rapporti che possono intercorrere tra infezione grippale e tubercolosi polmonare. Nella sua esperienza clinica non aveva trovato alcuna evidenza di maggiore disposizione all'infezione influenzale dei malati di tubercolosi.

\section{Conclusione}

Alcune coincidenze tristi e curiose precedettero o seguirono l'epidemia influenzale del 1918 a Catania, anche se non sono dipendenti o collegabili ad essa, neppure quando sono riportate sotto forma di notizie di cronaca nei giornali dell'epoca. La prima riguarda la morte del prof. Giovan Pietro Grimaldi a Modica il $1^{0}$ settembre 1918, già professore di fisica e rettore dell'Università, socio effettivo dell'Accademia; la seconda, l'eruzione laterale dell'Etna nel novembre 1918 (Giornale dell'Isola, anno V, n. 168, 22.06.1919) (De Fiore, 1920); la terza, l'esplosione violenta dello Stromboli il 22 maggio 1919 (Giornale dell'Isola, anno V, n. 146, 31.05.1919), la quarta la morte a Roma del prof. Annibale Riccò il 23 settembre 1919, presidente in carica dell'Accademia Gioenia. Le coincidenze sembrano sottolineare e contornare l'atmosfera inquieta e deflagrante del periodo, disseminato dagli strascichi sociali seguiti all'evento bellico appena conclusosi fortunatamente in modo vittorioso e dalla crisi economica da affrontare e risolvere. Le associazioni meta-scientifiche che accompagnano il sentimento della gente comune in difficoltà sanitaria sono sempre esistite. L'epidemia d'influenza sprigiona tutto il suo potenziale simbolico anche dopo che è riconosciuta come malattia, come è stato per la peste.

Notiamo che la pandemia di certo non catturò la sensibilità clinica dei soci accademici di cultura e professione medica. Possiamo trovare una debole giustificazione nel fatto che la maggior parte dei soci avevano attenzione e familiarità più verso le scienze naturali, la matematica e la fisica che verso la medicina. L'igienista prof. Eugenio Di Mattei seguiva e studiava al tempo episodi di peste a Catania (Di Mattei, 1923-1924), in scia a quelli già scoppiati alla fine dell'800. Il patologo medico Maurizio Ascoli e il patologo generale Giambattista Ughetti dedicavano i loro studi a malattie come la malaria, la tubercolosi, il tifo, il colera. Le basi anatomo-patologiche della pandemia e del confronto di dati e opinioni sull'eziologia della grande infezione sembrano dunque sfuggire al loro interesse, forse perché ebbero di fronte una malattia episodica, rapida, scomparsa in breve tempo così come era sopraggiunta in città e provincia nel settembre 1918. Nel primo semestre 1919 i giornali catanesi sono impegnati a raccontare gli ultimi avvenimenti militari e la convulsa politica post-bellica nazionale ed europea. Non riportano più informazioni a riguardo della pandemia influenzale, mentre sono prodighi di notizie di cronaca cittadina che riguardano la consegna di premi e medaglie agli eroi di guerra, il ritorno dei prigionieri, le prolusioni e inaugurazioni dell'anno accademico e dell'anno giudiziario, i cartelloni degli spettacoli nei teatri Massimo Bellini, Sangiorgi, Politeama Pacini e 
al cinema Olympia, il programma della festa di Sant'Agata. I lutti, le preoccupazioni per l'infezione e le misure igienico-sanitarie frettolosamente approntate tutto fu presto dimenticato.

\section{Bibliografia}

Aradas, S. (1919-1920). L'influenza. Note cliniche ed osservazioni critiche. Atti Acc. Gioenia serie V, vol. XII, Memoria VI.

Aymard, M. (1975). Epidémies et médecins en Sicile à l'époque moderne (Tipografia Fusi, Pavia).

De Fiore, O. (1920). L'eruzione radiale dell'Etna del novembre (?) 1918. Boll. Acc. Gioenia serie 2a, fasc. 48, 42.

de Quatrefages, J. L. A. (1857). The rambles of a naturalist on the coasts of France, Spain, and Sicily, translated by E. C. Otté, 2 voll. (Longman, Brown, Green, Longmans, \& Roberts, London).

Di Mattei, E. (1923-1924). L'episodio di peste in Catania dell'anno 1920. Atti Acc. Gioenia XIV, 1.

Fagiuoli, A. (1920). Sui rapporti tra tubercolosi e influenza. Boll. Acc. Gioenia serie 2a, fasc. 48, 26.

Izar, G. (1920). Acetonuria nell'influenza. Boll. Acc. Gioenia serie 2a, fasc. 48, 13.

Kolata, G. (2000). Epidemia: storia della grande influenza del 1918 e della ricerca di un virus mortale, p. 318 (Mondadori, Milano).

Maggiore Perni, F. (1894). Palermo e le sue grandi epidemie. Dal XVI al XIX secolo (Stabilimento tipografico Virzì, Palermo).

Oxford, J. S. (2001). The so-called Great Spanish Influenza Pandemic of 1918 may have originated in France in 1916. Phil. Trans. R. Soc. Lond. B 356(1416), 1857. doi:10.1098/rstb.2001.1012.

Petrone, A. (1918). Sulla cosidetta Febbre spagnuola. Boll. Acc. Gioenia serie 2a, fasc. 45,5 .

Previtera, S. (1919). Sull'epidemia d'influenza in Catania nel 1918. Boll. Acc. Gioenia serie $2 \mathrm{a}$, fasc. $46,37$.

Smith, W., Andrewes, C. H., and Laidlaw, P. P. (1933). A virus obtained from influenza patients. The Lancet 222(5732), 66. doi:10.1016/S0140-6736(00)785412.

Spinney, L. (2018). 1918. L'influenza spagnola. La pandemia che cambiò il mondo (Marsilio, Venezia).

Tognotti, E. (2015). La spagnola in Italia: storia dell'influenza che fece temere la fine del mondo (1918-19), prefazione di Giovanni Rezza, presentazione di Gilberto Corbellini (Angeli, Milano).

Vicentini, C. B., Guidi, E., silvia Lupi, Maritati, M., Manfedini, S., and Contini, C. (2015). L'influenza nelle ondate epidemiche del XIX secolo. The nineteenth-century epidemic waves of influenza. Infez. Med. 4, 374.

Winter, J. (2010). L'infuenza spagnola. In Audoin-Rouzeau, S. and Becker, J., editors, La prima guerra mondiale, page 288 (Einaudi, Torino). 